\title{
Mesure, par la méthode conductimétrique, de l'activité enzymatique de trois variétés de présure sur du lait frais de mélange et du lait reconstitué à partir de poudre dégraissée
}

\author{
par \\ J. TSOULI, G. FAVRE-BONVIN, Ch. POLICARD \\ et A. VILLE*
}

Plusieurs travaux ont été consacrés à l'étude de l'activité enzymatique de la présure ou de ses succédanés.

Soxhlet [1] définit la notion de "force " d'une présure largement utilisée pour les solutions commerciales.

Berridge [2] préconise pour sa part l'unité présure (U.P.) en faisant intervenir un substrat standard à base de lait écrémé en poudre.

Alais et al. [3] et Garnier [4] proposent l'étude de la quantité d'azote soluble dans l'acide trichloracétique 12 p. 100 ou la quantité de protons libérés à partir de la caséine $\chi$. Scott Blain et Oosthuizen [5] préconisent de suivre la chute de viscosité des solutions de caséinate de sodium.

D'après des travaux récemment effectués $[6,7,8]$ nous savons que la conductimétrie peut être utilisée pour suivre des cinétiques enzymatiques. Nous avons eu l'idée d'appliquer cette méthode pour mesurer l'activité enzymatique d'un mélange pepsine-présure, d'une présure d'origine fongique provenant de Mucor miehei et d'un extrait de fleur d'artichaut [9].

\section{MODALITES EXPERIMENTALES}

\section{1) Produits utilisés}

- Lait frais de mélange* $\mathrm{pH}$ 6,5-6,6.

- Lait reconstitué à partir de poudre dégraissée pH 6,5.

* Institut Universitaire de Technologie, Département de Biologie Appliquée, 43, boulevard du 11 Novembre 1918 - 69621 Villeurbanne.

* Nous tenons à remercier les responsables des Ets Rousset à Vénissieux pour avoir mis à notre disposition du lait frais et des préparations enzymatiques servant à leurs fabrications. 
- Mélange pepsine-présure* (50 p. 100 de pepsine de porc et 50 p. 100 de présure traditionnelle) ayant une force coagulante de $1 / 10000$.

- Extrait enzymatique provenant de Mucor miehei* ayant une force coagulante de $1 / 15000$.

- Extrait de fleur d'artichaut du genre Cinara cardunculus L ayant une force coagulante de l'ordre de 1/160.

\section{2) Appareillage}

Le montage a fait l'objet de travaux déjà cités $[6,7,8]$. Nous utilisons une cellule thermostatée à $35^{\circ} \mathrm{C}$ dont les électrodes sont portées par un agitateur axial. La constante de la cellule employée est d'environ $0,9 \mathrm{~cm}^{-1}$.

\section{3) Etude expérimentale}

$40 \mathrm{ml}$ de lait (soit frais, soit reconstitué à partir de poudre dégraissée) sont introduits dans la cellule et après stabilisation thermique à $35^{\circ} \mathrm{C}$ nous ajoutons $0,1 \mathrm{ml}$ d'une des solutions enzymatiques qui provoque instantanément un changement de conductivité très visible à l'enregistrement (la conductivité passe d'une valeur de l'ordre de $6,7 \mathrm{~m} \mho$ à $7,0 \mathrm{~m} \approx \boldsymbol{0})$. Ensuite après une légère augmentation, la conductivité subit une baisse sensible au moment précis de la " prise du lait ". Ce phénomène a été reproduit treize fois avec les deux sortes de lait et les trois variétés d'enzymes avec une fidélité remarquable.

Les courbes de la figure 1 représentent deux exemples de tracés obtenus par l'enregistreur au cours des manipulations effectuées.

Pour bien montrer que le point, à partir duquel nous assistons à une baisse de conductivité, correspond effectivement au début de la coagulation du lait, nous avons déterminé (parallèlement aux opérations précédentes et dans des conditions identiques) le temps de coagulation de l'enzyme employée [10].

Dès l'apparition des premiers flocons sur les parois du tube à essai, l'enregistrement est arrêté pour constater le même phénomène dans la cellule du conductimètre au moment où la courbe amorce un changement de pente.

\section{DISCUSSION}

La coagulation du lait est consécutive à une réaction protéolytique limitée dont la caséine $\chi$ est le substrat comme l'ont montré plusieurs travaux $[4,11,12,13,14,15]$. La caséine $\%$ est transformée 

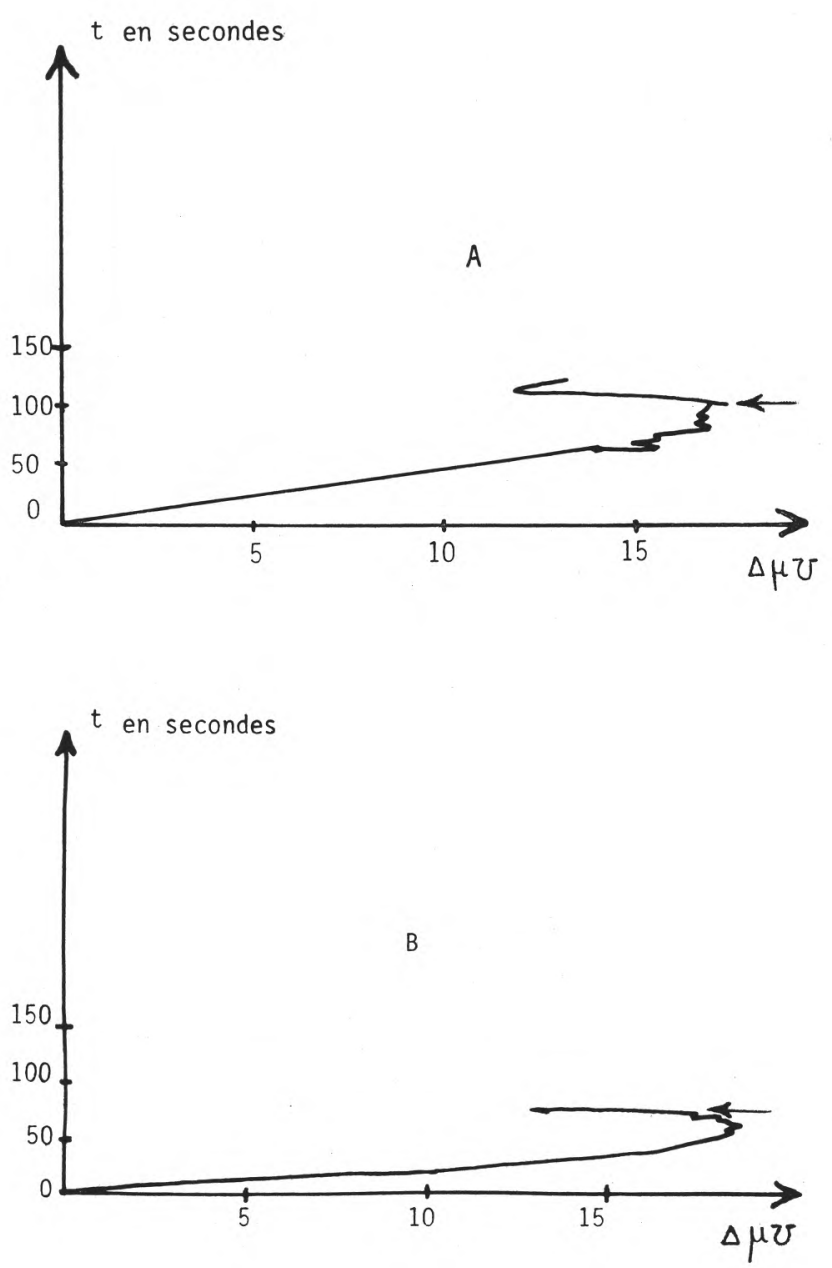

fig. 1

Variation de la conductivité $(\Delta \mu \mho)$ au cours de la coagulation du lait frais (1) ( $\mathrm{pH} \mathrm{6,6} \mathrm{;} \mathrm{température} \mathrm{d'emprésurage} 35^{\circ} \mathrm{C}$ ) en fonction du temps $(t)$ :

$1 \mathrm{~A}$, par le mélange pepsine-présure.

$1 \mathrm{~B}$, par l'extrait enzymatique provenant de Mucor miehei.

Les flèches sur les graphiques indiquent le moment à partir duquel se produit la coagulation.

(1) Les graphiques concernant la variation de la conductimétrie dans le cas du lait en poudre n'ont pas été reproduits car ils sont comparables à ceux de la figure 1 . 
en paracaséine $\chi$ qui forme un précipité fibrillaire rétractile insoluble de $\mathrm{pH} 4,6$ à $\mathrm{pH}>9$ mais soluble dans l'urée $6 \mathrm{M}$ ou à $\mathrm{pH}$ très alcalin [10]. Quant à la liaison hydrolysée, après avoir émis plusieurs hypothèses à son sujet : liaisons non covalentes, ester ou peptidique [16], on pense maintenant qu'il s'agit de liaison peptidique à la lumière de travaux récents [10].

Dans le présent travail, nous avons assisté au moment précis où se produit la coagulation à une chute de la conductivité du milieu ce qui se traduirait par une " indisponibilité " des ions calcium et phosphate et par suite une diminution des charges. Ceci rejoint les idées avancées par Alais [17] sur les conséquences de la séparation du caséine glycopeptide par la présure. Il est probable que d'autres éléments ioniques interviennent dans ce phénomène comme par exemple les autres sels minéraux (citrate, phosphate de calcium inorganique) qui, en dehors de leur rôle de complexant du calcium, semblent être indispensables dans la formation des micelles [18].

En outre, après avoir amorcé une chute au moment de l'apparition des premiers flocons, la conductivité connaît de nouveau une augmentation due probablement au phénomène déjà signalé par Garnier [4] à savoir la libération d'environ un proton pour trois monomètres de caséine $\%$ après la transformation de celle-ci en paracaséine.

La conductimétrie constitue donc une nouvelle méthode permettant de suivre la cinétique enzymatique de la présure ou de ses succédanés : de plus elle améliore la technique du temps de coagulation [10] : en effet grâce au déroulement régulier du papier d'enregistrement, nous pouvons apprécier, avec une précision remarquable, le temps écoulé entre le moment de l'injection de l'enzyme et celui de l'apparition des premiers flocons sans l'intervention du manipulateur : ainsi, dans le cas du mélange pepsine-présure (fig. $1 \mathrm{~A}$ ) nous pouvons lire un temps de coagulation de 1,6 $\mathrm{mn}$ et dans le cas de la présure d'origine fongique, ce temps est de 1,1 mn (fig. $1 \mathrm{~B}$ ).

D'autre part, grâce à cette méthode et en se mettant dans les conditions définies par Soxhlet [1], on peut trouver la force d'une présure donnée par la formule :

$$
\mathrm{F}=4 \times \frac{\mathrm{L}}{\mathrm{P}} \times \frac{1}{\mathrm{t}}
$$

dans laquelle : $\mathrm{L}=$ Volume de lait frais $; \mathrm{P}=$ Volume de la présure employée ; $\mathrm{t}=$ temps au bout duquel on obtient la coagulation (en $\mathrm{mn}$ ), le facteur temps étant obtenu ici avec une exactitude parfaite.

D'ailleurs quand on applique à la formule $F$ les valeurs que nous avons adoptées pour $\mathrm{L}$ et $\mathrm{p}$ et celle trouvée pour $\mathrm{t}$, on trouve : $F_{1}=40 \times \frac{40}{0,1} \times \frac{1}{1,1}=\frac{160000}{11} \simeq 14545$ pour la présure d'origine fongique, 
$\mathrm{F}_{2}=40 \times \frac{40}{0,1} \times \frac{1}{1,6}=10000 \quad$ pour le mélange pepsine-présure, c'est-à-dire pratiquement les mêmes valeurs qui nous ont été communiquées pour chacune des enzymes par les fournisseurs.

Dans la pratique, il est plus important de connaître le temps au bout duquel le caillé peut être travaillé, mais l'étude par la méthode conductimétrique du comportement du milieu après la " prise du lait » permettra peut-être d'apprécier ce temps précieux pour le fromager sans perturbation aucune du milieu biologique.

\section{Rés u m é}

L'étude de l'activité enzymatique de la présure ou de ses succédanés sur le lait par la méthode conductimétrique, montre que la conductivité subit une chute au moment précis où se produit la coagulation du lait.

Cette méthode permet d'apprécier aussi le temps de coagulation et par suite la «force » d'une présure telle qu'on la définit pour les solutions commerciales.

\section{S u m m a ry}

The conductrimetric method used for the study of the enzymatic activity of the rennet and its substitutes on milk, shows a falling of conductivity exactly at the milk-clotting.

The coagulation-time and consequently, the activity of the rennet, as it is defined for commercial solutions, is also estimated by this method.

Reçu pour publication en décembre 1974.

\section{Bibliographie}

[1] SoXhlet (F.) (1877). - Milchzeitung, 6, 495-501 et 513-514.

[2] Berridge (N. J.) (1945). - Biochemic J., 39, 179-186.

[3] Alais (C.), Mocouot (G.), Nitschmann (Hs.) et Zahler (P.) (1953). - Helv. Chim. Acta, 36, 1955-1968.

[4] Garnier (J.) (1963). - Ann. Biol. An. Bioch. Biophys., 3, 71-90.

[5] Scott-Blain (G. W.) et Oosthiuzen (J. C.) (1961). - J. Dairy Res., 28, 165-173.

[6] Policard (C.), Wallach (J.), Ville (A.), Hanss (M.) (1974). - Ann. Phys. Biol. et Med. (sous presse). 
[7] Policard (C.) (1972). - Thèse de troisième cycle Biochimie, Lyon.

[8] Hanss (M.) et Rey (A.) (1971). - Biochim. Biophys. Acta, 227, 618-629 et 630-638.

[9] Tsouli (J.) (1970). - C.R. Ac. Sciences, 270, 396-399.

[10] Garnier (J.), Mocouot (G.), Ribadeau-Dumas (B.), Maubois (J. L.). - Ann. Nutr. Alim., 22, B 495 - B 552.

[11] Garnier (J.) (1957). - Proc. Inst. Symp. Enzyme Chem., Tokyo, 524-526.

[12] WaKe (R. G.) (1957). - Aust. J. Sc., 20, 167.

[13] Waugh (D. F.), Von Hippel (P. H.) (1956). - J. Am. Chem. Soc., 78, 4576-4582.

[14] Nitschmann (Hs.) et Beeby - (1960). - Chimia, 14, 318-319.

[15] Jolles (P.) et Alais (C.) (1960). - Compt. Rend. Acad. Sci. Paris, 251, 6205-6207.

[16] Foltmann (B.) (1966). - Compt. Rend. Trav. Lab. Carlsberg, 35, 143-231.

[17] Alars (C.) (1965). - Sciences du lait, sept., 102-139.

[18] Waugh (D. F.). - 2e Cong. Int. de Biophys., Vienne 1966. Abst. 104, les An. de Phy. Biol et Méd. 